\title{
Hyperexpression of GAD in the islets may be relevant but is it sufficient to induce autoimmune insulitis?
}

\author{
P.Pozzilli ${ }^{1}$, R.D.G. Leslie ${ }^{2}$ \\ ${ }^{1}$ Endocrinologia I, University of Rome "La Sapienza", Rome, Italy \\ ${ }^{2}$ Department of Diabetes and Metabolism, St. Bartholomew's Hospital Medical College, London, UK
}

In their article, Drs. Degli Esposti and Mackay propose a mechanism whereby an islet antigen glutamic acid decarboxylase (GAD) could be hyperexpressed on islet cells, resulting in an aggressive immune attack on that cell with consequent destruction, loss of insulin secretory capacity and thus insulin-dependent diabetes mellitus (IDDM). It is our purpose to present briefly the many different mechanisms whereby self or non-self antigens might become targets of the immune response and progress to IDDM. Since the precise nature of the target antigen, if it exists, is unresolved, we think that the interesting mechanism proposed by Degli Esposti and Mackay, whereby stressinduced hyperexpression of GAD (and possibly other islet cell antigens) leads to IDDM, should be seen in a wider context as only one of many potential mechanisms which could cause this type of diabetes.

IDDM is believed to be due to an interaction of environmental and genetic factors [1]. Since genetic susceptibility to IDDM operates largely through the HLA region which encodes for proteins involved in antigen presentation and recognition, the immune response is probably important in the aetiology of the disease. The rapid destruction of apparently normal beta cells when transplanted from a non-diabetic twin to his/her identical diabetic twin [2] favours a cell-specific cytotoxic process with a long-standing memory, and an aberrant immune response seems to be the best candidate. Two theories have been proposed to explain the targeted cell destruction - a direct beta-cell specific process [3] or an indirect nonspecific process to which beta cells are sensitive [4].

Corresponding author: P. Pozzilli, M. D., Istituto II Clinica Medica, Policlinico Umberto I, University of Rome "La Sapienza", I-00161 Rome, Italy

A bbreviations: IDDM, Insulin-dependent diabetes mellitus; GAD, glutamic acid decarboxylase; NIDDM, non-insulin-dependent diabetes mellitus; GABA, $\gamma$-aminobutyric acid.
The first hypothesis envisages presentation of a betacell antigen to a T-cell receptor on CD4-positive $T$ cells in the context of an HLA molecule and the subsequent cascade of events leading to destruction of the cell expressing that target antigen [3]. According to the second hypothesis, macrophage and T-helper-cell-dependent mechanisms are involved in an inflammatory process which is recruited and amplified by T-helper cells [4]. This inflammatory process is elicited by an immune response to a self or non-self antigen either in the beta cell or in its vicinity, and beta-cell destruction is a consequence of increased cytokines in the beta-cell milieu.

\section{I slets at diagnosis of ID D M}

About $80 \%$ of islets contain no beta cells at the time of IDDM detection and the islets may be heavily infiltrated with mononuclear cells [5]. These cells include macrophages and antigen-specific CD8-positive T lymphocytes; the T cells show enrichment for certain $\mathrm{V} \beta$ T-cell receptors [6]. An increased expression of intercellular adhesion molecules on the vascular endothelium may enhance the accumulation of mononuclear cells in the islets [7]. However, lymphocyte infiltration is not a consistent feature, and occurs principally in the islets containing insulin-positive beta cells. Not all the insulin-secreting beta cells will be destroyed, and even in patients with long-standing diabetes about $10 \%$ of the islets can have insulincontaining cells [8]. Those residual islets which contain beta cells hyperexpress class I HLA antigens, but only the beta cells express class II HLA antigen and contain immunoreactive alpha-interferon [9]. The presence of alpha-interferon suggests that the cells have been chronically infected with a virus. However, there is no evidence to date that the beta cells contain viral components. Hyperexpression of 
class I or class II HLA antigens could also result from viral infection or, alternatively, it could be secondary to the inflammatory process. Islet infiltrating $\mathrm{T}$ cells from the pancreases of two children who died in diabetic ketoacidosis were examined for the T-cell receptor $\mathrm{V} \beta$ gene usage [6]. In both cases there was an expansion of the $\mathrm{V} \beta 7$ gene segment and normal peripheral blood $\mathrm{T}$ cells could be expanded, selecting the same gene segment, by membrane preparation from islets of patients but not control subjects. The ability to expand T cells selectively in this way is a hallmark of superantigens, small proteins produced by viruses and bacteria. More information is required regarding the sequence of changes which characterise islets during the prediabetic phase in order to interpret the significance of these changes.

\section{Induction of ID D M}

The most powerful evidence that immune-mediated diseases are due to non-genetically determined factors comes from the study of identical twins. As identical twins usually live together in childhood, similarities (concordance) between them might be due to shared genetic or non-genetic factors. Differences or discordance between identical twins, on the other hand, must be due to non-genetically determined factors. Most identical twin studies of autoimmune diseases demonstrate that the majority of co-twins of affected twins, including IDDM twins, do not develop the disease themselves. Discordance for autoimmune disease in monozygotic twins may be due to lack of, or difference in, exposure to antigens or differences in the immune response to antigens. Even identical twins can be genetically different for certain genes. For example, genes could undergo random and ordered rearrangement or mutation and may differ between identical twins. In contrast to antibody genes, genes of the Tcell receptor do not appear to undergo somatic hypermutation. If, as is widely believed, T cells are responsible for the immune destruction of beta cells then it is unlikely that somatic mutations give rise to IDDM. In addition, there is no evidence of randomness in either the clinical syndrome or the antigens recognised by antibodies in patients with the disease [10]. If a somatic mutation of the immune system causes IDDM, the aberrant immune response would probably be monoclonal and not polyclonal as is the case in this disease [11]. We therefore believe, though we cannot be certain, that the disease is due to environmental agents and not to a somatic mutation [12].

\section{A ntigen targets of autoimmunity}

In distinguishing between self and non-self, the organism's most efficient approach is to identify features (antigens) conserved within a species, and not features unique to a sub-type of that species. Therefore, conserved elements, both structural and functional, are obvious targets for immunity. Organspecific diseases tend to target functional elements such as enzymes, e.g. thyroid peroxidase (Hashimoto's thyroiditis) while non-organ-specific diseases target structural elements, e.g. histones and DNA (systemic lupus erythematosus). The 'luxury function' of all endocrine cells is to recognize a signal (glucose for the beta cell) and produce and secrete a hormone (insulin and insulin precursors). While IDDM patients can have antibodies which interfere with the cells' glucose transport [13], hormone secretion systems may be more important antigen targets and include enzymes (carboxypeptidase H, GAD, and protein tyrosine phosphatases), insulin and proinsulin. One of the striking features of antigens associated with IDDM is their sheer number. The existence of so many antigens obfuscates efforts to identify the single antigen but, at the same time, raises the possibility that many or all of the antigens are mere epiphenomena. Whatever the case, the critical antigens could be self-antigens or non-self antigens.

While there is an enormous number of potential antigenic determinants in any individual, only a minor fraction is actually presented to the immune system in an immunodominant manner. The remaining peptides are effectively silent, i.e. they are subdominant or cryptic. It remains to be determined why one epitope is selected to be immunodominant while the majority of other potential determinants are neglected. The hierarchy of display of dominant and cryptic determinants may shift and these shifts are dependent on a variety of extracellular factors including the inflammatory state of the milieu and the local antigen presenting cell population. Subtle changes in the antigen and how it is presented can determine whether immunity or tolerance to that antigen develops. It is this balance between tolerance and immunity which can be shifted, thereby altering susceptibility either to infections or to autoimmune diseases.

\section{N on-self antigen could be critical}

Evidence is limited that a non-self antigen, that is an environmental agent, is the target for the destructive immune response causing IDDM. In man, congenital rubella infection can cause diabetes of a similar type to both IDDM or non-insulin-dependent diabetes (NIDDM). Diabetes is found in about $20 \%$ of patients with the congenital rubella syndrome with a latent period of many years and a median age of onset of 13 years [14]. Other evidence, linking antibodies to viruses such as coxsackie virus at the time of diagnosis of diabetes, is less persuasive since at best it only implies that a virus precipitates the disease 
onset, and not that it induces the disease process. Nevertheless, viral infections may provoke an autoimmune disease, for example patients with subacute thyroiditis have a clinical picture consistent with an acute viral infection. Antibodies and T-cell reactivity to thyroid autoantigens can be detected in such patients and, while in the majority the autoimmune phenomena are transient, they can progress to autoimmune thyroid disease.

A second hypothesis suggests that an immune response directed against a non-self antigen, containing a peptide sequence homologous with a self-antigen, could activate an autoimmune process against that self antigen. This so-called 'molecular mimicry' hypothesis [15] receives support from studies of multiple sclerosis [16]. In that disease, patients' T-cell clones to the immunodominant antigen, myelin basic protein, can be activated by a variety of viral and bacterial peptides. In IDDM, an amino acid sequence in GAD within the region 247-279 is a major determinant with significant sequence similarity to a P2-C protein of the coxsackie $B$ virus $[17,18]$. Peripheral blood mononuclear cells from IDDM patients who respond to GAD peptides within the 247-279 region also responded to a homologous coxsackie viral peptide. Thus, molecular mimicry could lead to an altered immune response against self antigens even though the altered immune response was initially induced by non-self antigens.

Apart from viruses, toxins are the other main candidates implicated as environmental factors inducing IDDM. Of the many factors that have been proposed the most convincing evidence is that early exposure to cow's milk or a reduced duration of breast feeding in the first few months of life is associated with an increased disease risk [19]. Studies detecting antibodies to various components of cow's milk, including bovine serum albumin, have given conflicting results. More recent studies, however, have demonstrated an increased T-cell reactivity in IDDM patients to Beta casein [20] and to bovine beta lactoglobulin [21], but not to albumin or alpha casein. These T-cell studies need to be confirmed but they do raise the possibility that a substantial proportion of patients with recentonset IDDM have immunity to cow's milk proteins, in contrast to non-diabetic subjects. Interestingly, in line with the molecular mimicry hypothesis, there are five amino acids in bovine Beta casein which are identical to a glucose transporter (GLUT2), a glucose sensing protein in the islet beta cells.

\section{Self antigen could be critical}

Candidate autoantigens include tissue-specific proteins and glycolipids against which an immune response, such as autoantibodies, has been generated. These autoantigens include insulin, proinsulin, protein tyrosine phosphatases (ICA 512/IA-2 and IA- $2 \beta$ ) and GAD. Many studies have established the striking frequency of antibodies to these islet antigens in IDDM, as well as their predictive power both in the general population and in relatives of IDDM patients [22], but information on T-cell responses to these antigens is still at an early stage.

There is no evidence that these islet self-antigens have been structurally altered or mutated. Indeed, antigenic determinants or epitopes are highly conserved in evolution. It remains to be determined whether beta cells, that is islet self-antigens, are required for activation of the autoimmune response. Evidence that the expression of autoantigen in the target organ is required for the activation of autoreactive $T$ cells comes from the study of beta-cell deprived non-obese diabetic (NOD) mice. Following a toxic dose of alloxan the spleen cells from NOD mice can transfer diabetes into irradiated 8-week-old male NOD mice but not into 6-month-old beta-cell-deprived NOD mice [23]. Remarkably, both in NOD mice and in man the autoimmune destruction does not appear to affect other endocrine islet cells even though they contain GAD and the protein tyrosine phosphatase ICA512/IA-2. GAD, particularly the major isoform GAD $65 \mathrm{kDa}$, has emerged as a T-cell antigen associated with IDDM [24, 25]. In man, antibody and T-cell reactivity to GAD have been described both at the time of and prior to diagnosis and differences between IDDM patients and control subjects have been defined in studies of T-cell reactivity to short overlapping peptides [18]. IDDM patients have been shown to respond immunodominantly to peptides $473-555$ of GAD65 or preferentially to region 247279 of GAD65 in contrast to control subjects who respond mainly to the central region $[17,18]$. Such small antigen-specific differences between patients with IDDM and control subjects raise the possibility that the destructive T-cell response can be modulated by therapy to prevent IDDM.

\section{Implications for prevention}

IDDM can be regarded as a chronic destructive disease of the beta cells in which the destructive process is initiated at an early age by an environmental event. A primary prevention strategy for IDDM requires that critical environmental factors, for example cow's milk, are recognized and removed, or their effect negated. Primary intervention programmes in the general population or in high-risk groups might aim to eliminate or modify the response to these agents. Secondary prevention of IDDM would involve the identification of high-risk subjects, a feasible strategy given the evidence for a long prediabetic prodrome. Early identification of such subjects should enable us to intervene at a stage when the residual beta-cell 
mass is sufficient to sustain normoglycaemia. It is possible that some natural regulatory system may, in some cases, limit progression to the disease since many of the immune and metabolic changes associated with IDDM can occur without leading to clinical diabetes. Tilting the balance of this immune regulation away from destruction might be sufficient to prevent the onset of diabetes.

The strategies that could be envisaged include non-specific agents such as nicotinamide or antigenspecific agents such as insulin. Many potential antigen-specific interventions to prevent diabetes have been used in animal models of the disease [26]. One strategy is to reduce beta-cell antigen expression. There is evidence for increased metabolic activity in prediabetic beta cells both in man and in the NOD mouse, i. e., the existence of fasting hyperproinsulinaemia in man and of hyperinsulinaemia in NOD mice. As a result of these observations one study attempted to down-regulate GAD expression with a view to preventing or reducing the frequency of diabetes. Baclofen, a gamma-aminobutyric acid (GABA)- $\beta$ receptor agonist, was administered to NOD mice from an early age [27]. In NOD mice baclofen did delay, but did not prevent, the onset of diabetes. However, baclofen did not down-regulate GAD activity sufficiently to block the autoimmune process at the doses used. Thus, either a slight reduction in GAD or modulation of GABA activity in the islets may interfere with the process leading to betacell destruction and clinical diabetes; greater reductions may be sufficient to prevent disease progression. Alternatively, as GABA has been detected in the beta cells, where it is localized in a population of synaptic-like microvesicles [28] and secreted in a regulated fashion, baclofen may modulate GABA secretion from the islets, by suppressing other antigens, including insulin.

\section{Conclusion}

We have outlined the many different ways an autoaggressive beta-cell response could be induced and maintained. Relevant antigens could be self- or nonself antigens which may or may not be abnormally presented to normal or abnormal $\mathrm{T}$ cells before the destructive T-cell response is mounted. That downregulation of GAD may influence diabetes outcome in NOD mice suggests that GABA activity could be one relevant factor in the generation of an aggressive immune response towards beta cells. The hypothesis of Degli Esposti and Mackay relating to hyperexpression of GAD would be consistent with the findings reported in NOD mice. However, such a hypothesis must be regarded as one of many interesting mechanisms which could cause autoimmunity in general and IDDM in particular.

\section{References}

1. Eisenbarth G (1986) Type 1 diabetes mellitus. N Engl J Med 314: 1360-1368

2. Eisenbarth GS, Stegall M (1996) Islet and pancreatic transplantation - autoimmunity and alloimmunity. N Engl J Med 335: 888-890

3. Atkinson MA, Maclaren NK (1994) Mechanisms of disease: the pathogenesis of insulin-dependent diabetes mellitus. N Engl J Med 331: 1428-1436

4. Mandrup-Poulsen T (1996) The role of interleukin-1 in the pathogenesis of IDDM. Diabetologia 39: 1005-1029

5. Pipeleers D, Ling Z (1992) Pancreatic beta cells in insulindependent diabetes. Diab Metab Rev 8: 209-227

6. Conrad B, Weidmann E, Trucco G et al. (1994) Evidence for superantigen involvement in insulin-dependent diabetes mellitus etiology. Nature 371: 351-355

7. Hanninen A, Jalkanen S, Salm M et al. (1992) Macrophages, T cell receptor usage, and endothelial cell activation in the pancreas at the onset of insulin-dependent diabetes mellitus. J Clin Invest 90: 1901-1910

8. Foulis AK, Liddle CN, Farquharson MA, Richmond JA, Weir RS (1986) The histopathology of the pancreas in type 1 (insulin-dependent) diabetes mellitus: a 25-year review of deaths in patients under 20 years of age in the United Kingdom. Diabetologia 29: 267-274

9. Foulis AK, Farquharson MA, Hardman R (1987) Aberrant expression of class II major histocompatibility complex molecules by $\beta$ cell and hyperexpression of class I major histocompatibility complex molecules by insulin containing islets in type 1 (insulin-dependent) diabetes mellitus. Diabetologia 30: $333-343$

10. Harrison LC (1994) Islet cell antibodies. Light at the end of the tube? Diabetes Care 17: 1220-1223

11. Millward A, Hussain MJ, Peakman M, Pyke DA, Leslie RDG, Vergani D (1988) Characterization of islet cell antibody in insulin dependent diabetes: evidence for IgG1 subclass restriction and polyclonality. Clin Exp Immunol 71: 353-356

12. Leslie RDG, Elliott RB (1994) Early environmental events as a cause of IDDM: evidence and implications. Diabetes 43: $843-850$

13. Inman LR, McAllister CT, Chen L et al. (1993) Autoantibodies to the GLUT-2 glucose transporter of $\beta$ cells in insulin-dependent diabetes mellitus of recent onset. Proc Natl Acad Sci USA 90: 1281-1284

14. Forrest JM, Menser MA, Burgess JA (1971) High frequency of diabetes mellitus in young adults with congenital rubella. Lancet 2: 332-334

15. Baum H, Davies H, Peakman M (1996) Molecular mimicry in the MHC: hidden clues to autoimmunity? Immunol Today 17: 64-70

16. Brocke S, Gijbels K, Allegretta M et al. (1996) Treatment of experimental encephalomyelitis with peptide analogue of myelin basic protein. Nature 379: 343-346

17. Atkinson MA, Bowman MA, Campbell L, Darrow BL, Kaufman DL, Maclaren NK (1994) Cellular immunity to an epitope common to glutamate decarboxylase and coxsackie virus in insulin-dependent diabetes. J Clin Invest 94: $2125-2129$

18. Lohmann T, Leslie RDG, Hawa M, Geysen M, Rodda S, Londei M (1994) Immunodominant epitopes of glutamic acid decarboxylase 65 and 67 in insulin-dependent diabetes mellitus. Lancet 343: 1607-1608

19. Gerstein HC (1994) Cow's milk exposure and type 1 diabetes mellitus. A critical overview of the clinical literature. Diabetes Care 17: 13-19 
20. Cavallo MG, Fava D, Monetini L, Barone F, Pozzilli P (1996) Cell-mediated immune response to $\beta$ casein in recent-onset insulin-dependent diabetes mellitus: implications for disease pathogenesis. Lancet 348: 926-928

21. Vaarala O, Klemetti P, Savilhati E, Reijonen H, Ilonen J, Akerblom HK (1996) Cellular immune response to cow's mild beta-lactoglobulin in patients with newly-diagnosed IDDM. Diabetes 45: 178-182

22. Bingley PJ, Gale EAM (1996) Current status and future prospects for prediction of IDDM. In: Palmer JP (ed) Prediction, prevention and genetic counseling in IDDM. John Wiley and Sons Ltd., Chichester, pp 228-253

23. Larger E, Becourt C, Bach JF et al. (1995) Pancreatic islet beta cells drive $\mathrm{T}$ cell immune response in the non-obese diabetic mouse model. J Exp Med 181: 1635-1642

24. Atkinson MA, Kaufman DL, Campbell L et al. (1992) Response of peripheral-blood mononuclear cells to glutamate decarboxylase in insulin-dependent diabetes. Lancet 339: 458-459

25. Harrison LC, Honeyman MC, DeAizpurua HJ et al. (1993) Inverse relation between humoral and cellular immunity to glutamic acid decarboxylase in subjects at risk of insulindependent diabetes. Lancet 341: 1365-1369

26. Pozzilli P (1996) Prevention of insulin-dependent diabetes: where are we now? Diab Metab Rev 12: 127-136

27. Beales PE, Hawa M, Williams AJK, Albertini MC, Giorgini A, Pozzilli P (1995) Baclofen, a gamma-aminobutyric acid-b receptor agonist, delays diabetes onset in the nonobese diabetic mouse. Acta Diabetol 32: 53-56

28. Sorenson SL, Garry DG, Brelje TC (1991) Structural and functional considerations of GABA in islets of Langerhans. $\beta$-cells and nerves. Diabetes 40: 1365-1374 